\title{
In the nickel of time
}

\author{
Although reports on the use of nickel can be traced back to 3,500 BC, Catherine Drennan points to a \\ resurgence of interest in nickel-based chemistry in the energy and environmental areas.
}

$\mathrm{N}$ ickel is not new. Those interested in the origins of life have postulated that early life forms could have used mixtures of nickel, iron and sulfur to harvest energy from their environment rich in carbon dioxide, carbon monoxide and hydrogen ${ }^{1}$. Nickel's unintentional use by humans has been traced back as far as 3,500 BC in Syrian bronzes, although it was not formally recognized as an element until 1751.

Most Americans know that one use of nickel is as an ingredient in a coin - the 'nickel' to be exact. Although the face value of a nickel has always been five cents, the amount of nickel actually present in the coin has varied over the years between $25 \%$ of the content (of the original and current coins) to none at all in the 'wartime nickels'. Nickel is also found in stainless steel and rechargeable batteries, is used for catalytic hydrogenation, and can give a green tint to glass.

Nickel has several important properties that allow for these varied uses. It is one of the $d$-block transition metals, and is located between the pairs of iron and cobalt to the left, and copper and zinc to the right. Because of their $d$ electrons, transition metal complexes can be visually stunning, exhibiting a vast array of brilliant colours. These complexes can undergo large changes in oxidation (nickel oxidation states range from 0 to +4 ), display a wide variety of geometries, and form diverse types of bonds including organometallic ones with carbon atoms.
Like its nearest neighbours in the periodic table, nickel is very important in biology. It is essential to the survival of many microorganisms, including beneficial microbes that live in our intestines. Unfortunately, it also serves harmful microbes, for example strains of Escherichia coli that are responsible for bladder infections, through a protein known as nickel-iron hydrogenase, which interconverts dihydrogen with two protons and two electrons - a reaction involved in cellular energy production. Helicobacter pylori, which is associated with stomach ulcers, is able to live in the acidic conditions of the stomach thanks to a nickel-containing protein called urease, which breaks down urea into ammonia and carbon dioxide, neutralizing the acidity. Drugs that target the nickel uptake by $H$. pylori are therefore being investigated for ulcer therapies.

Another fascinating role of nickel in biology is found in the global carbon cycle. Although we generally think of carbon monoxide, carbon dioxide and methane as pollutants and greenhouse gases, some microbes see them as a source of energy. The term global carbon cycle refers to the fact that one microbe's fuel is another microbe's waste product. For example, phototropic anaerobic bacteria 'eat' carbon monoxide and produce carbon dioxide, whereas microbes called acetogens consume carbon dioxide and produce acetate. Meanwhile, methanogens live on acetate and generate methane. Methane subsequently enters the atmosphere, where it can be converted back to carbon dioxide through anaerobic degradation, thus completing the cycle.
At the heart of this carbon cycle are three nickel-containing enzymes: carbon monoxide dehydrogenase, acetyl-CoA synthase, and methyl-CoM reductase. Each year, the combined action of these three enzymes is estimated to be responsible for removing $10^{8}$ tons of carbon monoxide from the environment ${ }^{2}$ and producing $10^{11}$ tons of acetate $^{3}$ and $10^{9}$ tons of methane $e^{4}$. The ability of nickel to form bonds with carbon is key to this reactivity. It is also interesting to note that the proposed involvement of nickel in the primordial soup stems from microbes' ability to use it to live on carbon monoxide and carbon dioxide - gases that were abundant early in Earth's history.

Today, scientists are more interested in applying these nickel-containing enzymes to solve the world's current energy and environmental problems - hydrogenase chemistry is attractive for hydrogen fuel cell technology ${ }^{5}$, whereas the reactivity of carbon monoxide dehydrogenase and acetyl-CoA synthase could be used in coal plants to lower carbon dioxide emissions ${ }^{6,7}$. With issues such as global warming, urban pollution and the recent oil spill disaster in the gulf coast, we must be aware of how our quest for energy impacts the environment as we search for alternative energy sources. 'An oldie but a goodie', nickel chemistry may be in the nick of time.

\section{CATHERINE L. DRENNAN is a Howard} Hughes Medical Institute Professor and Investigator at Massachusetts Institute of Technology, 77 Massachusetts Avenue Cambridge, Massachusetts 02142, USA. e-mail: cdrennan@mit.edu

\section{References}

1. Wächtershäuser, G. \& Huber, C. Science 276, 245-247 (1997).

2. Bartholomew, G. W. \& Alexander, M. Appl. Environ. Microbiol. 37, 932-937 (1979).

3. Drake, H. L., Daniel, S. L., Matthies, C. \& Kusel, K. in Acetogenesis (ed. Drake, H. L.) 3-60 (Chapman and Hall, 1994).

4. Thauer, R. K. Microbiology 144, 2377-2406 (1998)

5. Posewitz, M. C., Mulder, D. W. \& Peters, J. W. Curr. Chem. Biol. 2, 178-199 (2008).

6. Stauffer, N. Energy Futures 6-8 (Autumn, 2009)

7. http://www.teachersdomain.org/resource/biot09.biotech.car.drennan/ 